\title{
Modelo de análisis para canales de YouTube: aplicación a medios nativos digitales
}

\section{Analysis Model for YouTube Channels: Application to Digital Native Media}

\author{
José Sixto-García ${ }^{1}$. Universidad de Santiago de Compostela. España \\ jose.sixto@usc.es \\ $[\mathrm{CV}] \bigcirc \mathrm{G}$
}

Ana Isabel Rodríguez-Vázquez. Universidad de Santiago de Compostela. España anaisabel.rodriguez.vazquez@usc.es

[CV] G

Xosé Soengas-Pérez. Universidad de Santiago de Compostela. España

jose.soengas@usc.es

[CV] $\mathrm{G}$

Este artículo se ha elaborado en el marco del proyecto Cibermedios nativos digitales en España: formatos narrativos y estrategia móvil (RTI2018-093346-B-C33), del Ministerio de Ciencia, Innovación y Universidades, cofinanciado por el Fondo Europeo de Desarrollo Regional (FEDER).

\section{Cómo citar este artículo / Referencia normalizada}

Sixto-García, J., Rodríguez-Vázquez, A. I. y Soengas-Pérez, X. (2021). Modelo de análisis para canales de YouTube: aplicación a medios nativos digitales. Revista Latina de Comunicación Social, 79, 1-16. https://www.doi.org/10.4185/RLCS-2021-1494

\section{RESUMEN}

Introducción y metodología: Se investiga la participación en YouTube de una muestra internacional de periódicos nativos digitales de referencia a partir de un modelo de análisis propuesto por los autores. Discusión: La evaluación tiene en cuenta las variables que caracterizan los modelos periodísticos de producción y distribución de contenidos en los escenarios convergentes actuales tales como la implementación de estrategias de cross-promotion, el empleo de narrativas transmedia o la habilitación de comunidades de usuarios o de espacios específicos para la cocreación. Resultados y conclusiones: Los resultados diagnostican un sistema de trabajo fundamentado en rutinas productivas basadas en la difusión multiplatorma y crossmedia donde, por una parte, se evidencian las fortalezas que YouTube ofrece para los medios de comunicación y, por otra, se destapan carencias significativas que repercuten directamente en los niveles de engagement con los públicos y en una disminución del tráfico web, de la cantidad de suscriptores de los canales y del número de visualizaciones de los vídeos.

PALABRAS CLAVE: YouTube; medios nativos digitales; canales; modelo de análisis; engagement; cross-promotion; crossmedia.

\footnotetext{
${ }^{1}$ Autor de correspondencia.
} 


\begin{abstract}
Introduction: The participation in YouTube of an international sample of reference digital native media is analyzed based on a model proposed by the authors. Methodology: The evaluation takes into account the variables that characterize the journalistic models of content production and distribution in current convergent scenarios, such as the implementation of cross-promotion strategies, the use of transmedia narratives or the empowerment of user communities or specific spaces for co-creation. Results and Conclusions: The results diagnose a work system based on productive routines focused on crossmedia. On the one hand, the strengths that YouTube offers for the media are evident, but, on the other hand, we found significant deficiencies that directly impact on the levels of engagement and in a decrease in web traffic, the number of subscribers to the channels and the number of views of the videos.
\end{abstract}

KEYWORDS: Youtube; digital native media; channels; analysis model; engagement; crosspromotion; crossmedia.

\title{
CONTENIDO
}

1. Introducción. 2. Objetivos. 3. Metodología. 4. Resultados. 5. Discusión y Conclusiones. 6. Referencias.

\section{Introducción}

Hace aproximadamente una década que las redes sociales se han convertido en un elemento necesario e imprescindible en la gestión de la comunicación organizacional (Sánchez, 2010). Desde la publicación del primer vídeo, 'Meet at the zoo' en 2005, YouTube ha ido incorporando cada vez más funcionalidades propias de red social, si bien existió un debate ya superado acerca de si era o no una red social. YouTube fue considerado un servicio para compartir vídeos (Lange, 2007), un medio para la distribución de contenidos generados por los usuarios (Zink et al., 2008), una red social (Haridakis \& Hanson, 2009), una comunidad de contenido (Smith, Fischer \& Yongjan, 2012) y un medio participativo (Burgess \& Green, 2013). A día de hoy YouTube puede considerarse una red social, aunque presenta características singulares con respecto a las demás tanto en el producto que ofrece (solo vídeo) como en la forma de consumirlo (el usuario ya prevé el producto que va a encontrar) y en el modo de acceder a él (no se exige registro ni configuración de perfil para acceder a los contenidos) (Sixto, 2018).

Waters y Jones (2011) explicaron el auge y el éxito del vídeo en la gestión de la comunicación al entender que proporcionan al usuario las tres Vs de la comunicación (verbal, vocal y visual), por lo que el nivel de impacto aumenta en varios frentes comunicativos y la marca se humaniza. Son varios los estudios previos que confirman la utilización de YouTube en las estrategias de gestión empresarial. Así, por ejemplo, un análisis realizado en la Eurozona demostró que el $44 \%$ de las empresas utilizaban esta red social (Bonsón, Bednarova \& Escobar-Rodríguez, 2014), otro que 16 de las 20 empresas españolas mejor reputadas según Merco contaban con un canal (Costa-Sánchez, 2014) u otro que sitúa a YouTube como la tercera red social más utilizada por las superbrands portuguesas (Costa-Sánchez, 2016). Con todo, siempre es cometido y responsabilidad del community manager decidir y valorar (Battaglini, 2015; Hernández, Silva \& Rivera, 2013) si la organización es capaz de nutrir y mantener el canal de YouTube (Hoyos \& Lasso de la Vega, 2017), pues es preferible la ausencia que una presencia desactualizada o mal gestionada. 
En la revisión de la literatura académica también hemos encontrado varias investigaciones que demuestran la aplicación de YouTube en sectores y ámbitos diversos como la política (Berrocal, Campos \& Redondo, 2012; Berrocal, Martín \& Gil, 2017; Gil, 2019; Gómez \& López, 2016), la moda (Díez, 2017), el activismo social (García, 2013), la maternidad (Curbelo, 2015) o el aprendizaje de idiomas (Asensio, 2018). Artero (2010) confirmó que YouTube tiene un alto poder de convocatoria para los usuarios, mientras que Shay y Van Der (2019) midieron el valor de las respuestas de las audiencias a las publicaciones en redes sociales iniciadas por las empresas y concluyeron que tienen una relación positiva con los valores de marca, a pesar de que matizan que publicar contenido en exceso puede tener un efecto adverso en dichos valores y reducir, en consecuencia, el alcance acumulativo de una organización.

Con respecto al ámbito concreto de los medios de comunicación, Cobo y Pardo (2007) comprobaron que fue a partir de las transformaciones aparecidas a raíz de la consolidación de la web 2.0, y por tanto de los medios y redes sociales, cuando los medios de comunicación tradicionales se vieron obligados a reconstruir su arquitectura y a ofrecer más recursos digitales. Carbajal (2008) analizó el impacto de YouTube en las webs de las televisiones españolas y Ruivo y Gómes-Franco (2019) sintetizaron cómo la agencia EFE refuerza su imagen de marca mediante un canal dirigido a la audiencia final, una alternativa por la que también apostó Televisión Española para difundir el archivo histórico (Desiderata, 2019).

Como los públicos se han fragmentado y se han convertido en sociales debido a la interactividad que practican en redes en paralelo al consumo que realizan de forma tradicional (Deltetll, 2014), los medios también se han visto obligados a adaptar su discurso digital a esos nuevos modelos de comportamiento y hábitos de consumo (Bazanini et al., 2015). El uso de las redes sociales favorece el tránsito del periodismo de una actividad de un solo canal a un sistema multicanal, de modo que tanto las empresas como los periodistas deben operar simultaneamente en varios canales en todas las fases de producción y distribución de noticias (Neuberger, Nuernbergk \& Langenohl, 2018).

Esta distribución multicanal y multiplataforma que empezaron a experimentar los medios, provocada a su vez por la recepción multipantalla a la que se habituaron los públicos, debe enmarcarse en los procesos de convergencia que afectaron a los medios desde finales de la década de 1990 y que repercutieron de manera directa en las empresas, en las tecnologías, en los profesionales y en las audiencias, y en cada una de las fases de producción, distribución y consumo de contenidos. Por tanto, la convergencia periodística debe entenderse como un proceso de integración de los modos de comunicación tradicionalmente separados que incide en las estrategias empresariales, en los cambios tecnológicos, en la elaboración y distribución de contenidos multiplataforma, en el perfil profesional de los periodistas y en las formas de acceder a los contenidos (Salaverría, García Avilés \& Masip, 2010).

En ese contexto convergente los medios empezaron a implementar estrategias narrativas específicas para adecuar la distribución de los contenidos a las necesidades de las audiencias y explotar las potencialidades que ofrecía la tecnología, entre las que destacamos las prácticas crossmedia y más recientemente las propuestas de cocreación de contenidos. En el primer caso, se trata de integrar diferentes canales, soportes o medios para lanzar un mensaje común, pero adaptado a los lenguajes específicos de cada plataforma, de tal manera que esa integración de contenidos de medios cruzados o convergentes proporciona información complementaria a los públicos (Ma, Tanaka \& Nadamoto, 2006) que incitan al usuario a la navegación de unos espacios a otros y cuya existencia puede estar publicitada mediante mecanismos de cross-promotion (Copple, 2012). En el caso de los procesos de cocreación la implicación de los usuarios es mucho mayor al permitirles coconstruir experiencias propias y singulares para cada uno de ellos gracias a la habilitación de espacios participativos 
(Prahalad \& Ramaswamy, 2004) que implican una modificación del storyboard y del storytelling originales. Pero también la tecnología, las redes sociales y movimientos como la gamificación, la social TV o la generalización de utilización de la segunda pantalla han contribuido a enriquecer el discurso y a facilitar la construcción de narrativas transmedia (Rivera, 2012) que suponen el fraccionamiento de las historias y, por tanto, la exigencia al usuario de visitar varios espacios para conseguir una perspectiva integral del relato.

Si a todo ello se suma que los recursos audiovisuales aumentan la credibilidad y la confianza de las marcas porque permiten corroborar los hechos (Wardle \& Derakhshan, 2017), y que otras investigaciones anteriores han confirmado la preferencia de las audiencias por los contenidos audiovisuales (Blanco \& Palomo, 2019), nos preguntamos si los medios más recientes como los cibermedios, creados en y para Internet, se concibieron desde una óptica convergente y apoyados en las redes sociales para la distribución de contenidos. En concreto, se pretende observar si recurren a YouTube con el objetivo de enriquecer audiovisualmente el discurso textual favoreciendo la verificación de los hechos. Recordemos, además, que la red social acrecentó en 2018 su compromiso con el periodismo de calidad al promocionar fuentes informativas de referencia y recomendar ese tipo de contenidos para evitar la desinformación (Lapowsky, 2018).

\section{Objetivos}

El Informe Digital 2020 publicado por We Are Social en colaboración con Hootsuite indica que el número de usuarios de redes sociales en el mundo ha superado ya la marca de los 3.8 billones y que YouTube se consolida como la segunda red social más usada del planeta con 2.000 millones de usuarios, solo superada por Facebook, con una audiencia de 2.449 millones de personas. Los datos avalan y justifican la necesidad de emplear YouTube en el discurso periodístico, pero iniciamos la investigación con la hipótesis de que son pocos los medios que hacen difusión continua de noticias en YouTube. Esta práctica, en cambio, es frecuente en Facebook, donde las páginas de los periódicos se caracterizan por una publicación constante y permanente de contenidos de actualidad.

El objetivo principal de esta investigación es analizar la presencia corporativa de los medios nativos digitales en YouTube. El examen se fundamenta a partir de los parámetros que se establecen en los siguientes objetivos secundarios:

a) Cuantificar el número de suscriptores, vídeos y visualizaciones de cada canal.

b) Identificar las frecuencias de publicación en el canal y en las comunidades, en caso de existir.

c) Reconocer estrategias de cross-promotion cuya existencia puede estar publicitada mediante mecanismos que incitan al usuario a la navegación de unos espacios a otros (Copple, 2012), es decir, entre web, redes sociales y espacios externos.

d) Registrar modelos de difusión de contenidos mediante narrativas crossmedia y transmedia.

e) Comprobar la explotación de recursos propios de YouTube para mejorar el engagement con los usuarios.

\section{Metodología}

La selección de la muestra se realiza con perspectiva internacional; se eligen un medio español, siete europeos y otro estadounidense (ver tabla 1), todos ellos nativos digitales de referencia en el contexto mundial y en sus respectivos países por la fuerza con que se adentraron en el escenario digital. Además, todos los medios componen la muestra del proyecto Cibermedios nativos digitales en España: formatos narrativos y estrategia móvil (RTI2018-093346-B-C33), del Ministerio de Ciencia, Innovación y Universidades, cofinanciado por el Fondo Europeo de Desarrollo Regional (FEDER), que financia esta investigación. 
Tabla 1. Muestra de medios analizados

\begin{tabular}{ll}
\hline \multicolumn{1}{c}{ Medio nativo digital } & \multicolumn{1}{c}{ País } \\
\hline Eldiario.es & España \\
\hline Les Jours & Francia \\
\hline De Correspondent & Países Bajos \\
\hline The Canary & Reino Unido \\
\hline Observador & Portugal \\
\hline Krautreporter & Alemania \\
\hline The Texas Tribune & Estados Unidos \\
\hline Il Post & Italia \\
\hline
\end{tabular}

Fuente: Elaboración propia

La selección responde, pues, a una muestra de carácter no probabilístico que se utiliza en escenarios donde la población es variable y pequeña, por lo que es pertinente (Igartua, 2006) seleccionar casos característicos de ese universo (López-Roldán \& Fachelli, 2015; Otzen and Manterola, 2017). Se comprueba, además, la presencia de esos medios en el Reuters Institute Digital News Report 2019 y, aunque los resultados (ver tabla 2) muestran que tres de estos nativos digitales se encuentran ya entre los medios con mayor porcentaje semanal de búsquedas y uso (\% weekly use), consideramos que YouTube puede ayudar al conjunto de medios a mejorar su posición en los respectivos contextos nacionales donde predominan las presencias de medios tradicionales o matriciales.

Tabla 2. Presencia de nativos digitales en Digital News Report (2019)

\begin{tabular}{llll}
\hline Medio nativo digital & $\begin{array}{c}\text { Presencia en } \\
\text { Digital News } \\
\text { Report 2019 }\end{array}$ & $\begin{array}{c}\text { Posición en país en } \\
\text { cuanto a \% } \\
\text { semanal de uso }\end{array}$ & $\begin{array}{c}\text { Brand Trust Scores } \\
\text { (0-10) } \\
\text { (personas que han } \\
\text { escuchado hablar } \\
\text { sobre la marca) }\end{array}$ \\
\hline Eldiario.es & $\mathrm{X}$ & $4 / 16$ & - \\
\hline Les Jours & - & - & - \\
\hline De Correspondent & - & - & 5.91 \\
\hline The Canary & $\mathrm{X}$ & $19 / 20$ & 4.72 \\
\hline Observador & $\mathrm{X}$ & $8 / 16$ & 6.38 \\
\hline Krautreporter & - & - & - \\
\hline The Texas Tribune & - & - & - \\
\hline Il Post & - & - & - \\
\hline
\end{tabular}

Fuente: Elaboración propia

Para el análisis de las presencias se diseñó una ficha compuesta por nueve ítems de evaluación que dan respuesta a los objetivos de investigación planteados (ver tabla 3) y que se aplicó al último año (de mayo 2019 a mayo 2020). En la investigación se combinaron técnicas metodológicas de carácter cuantitativo (frecuencias de publicación o número de suscriptores) con otras de carácter cualitativo (análisis de contenido u observación directa desde la perspectiva de usuario). A cada ítem se le asignaron las diferentes variables de implementación y a cada una de ellas una puntuación en función del nivel de consecución. Para el establecimiento de puntuaciones tomamos como referencia el modelo de las escalas de Likert (Wigley, 2013), en el sentido de que se establecen escalas donde la puntuación más alta se reserva a la excelencia y la más baja a situaciones mejorables. 
Cada ítem se valora hasta 1 punto. Todas las variables se aplican de modo excluyente entre sí salvo en el caso de las posibilidades o recursos de difusión, donde la utilización de podcasts suma 0,25 puntos, pero no se incluye en las variables anteriores no por su singularidad, sino por tratarse de un formato que no es común en YouTube y, por tanto, que no procede exigir. La obtención de la puntuación máxima en todos los ítems suma 12 puntos (12,25 puntos si se tienen en cuenta los podcasts).

Tabla 3. Modelo de análisis de canales de YouTube

\begin{tabular}{|c|c|c|c|}
\hline & \multirow{5}{*}{$\begin{array}{r}\text { Ítem de valoración } \\
\text { Frecuencia de publicación }\end{array}$} & Variables & \multirow{2}{*}{1} \\
\hline & & Diaria & \\
\hline & & Semanal & 0,5 \\
\hline & & Mensual & 0,1 \\
\hline & & $>$ Mensual & 0 \\
\hline \multirow[t]{6}{*}{ Comunidades } & \multirow[t]{2}{*}{ Habilitación de comunidad } & Sí & 1 \\
\hline & & No & 0 \\
\hline & \multirow{4}{*}{$\begin{array}{l}\text { Frecuencia de publicación en } \\
\text { comunidad }\end{array}$} & Diaria & 1 \\
\hline & & Semanal & 0,5 \\
\hline & & Mensual & 0,1 \\
\hline & & $>$ Mensual & 0 \\
\hline \multirow[t]{10}{*}{ Cross-promotion } & \multirow{2}{*}{$\begin{array}{l}\text { Cross-promotion de web a } \\
\text { YouTube }\end{array}$} & Sí & 1 \\
\hline & & No & 0 \\
\hline & \multirow{2}{*}{$\begin{array}{l}\text { Cross-promotion de YouTube a } \\
\text { web }\end{array}$} & Sí & 1 \\
\hline & & No & 0 \\
\hline & \multirow{2}{*}{$\begin{array}{l}\text { Cross-promotion de YouTube a } \\
\text { otras redes o apps }\end{array}$} & Sí & 1 \\
\hline & & No & 0 \\
\hline & \multirow{4}{*}{$\begin{array}{l}\text { Cross-promotion mediante } \\
\text { canales externos }\end{array}$} & Sí (>10) & 1 \\
\hline & & Sí (>1) & 0,5 \\
\hline & & Sí (1) & 0,25 \\
\hline & & No & 0 \\
\hline \multirow{9}{*}{$\begin{array}{l}\text { Difusión, } \\
\text { recursos } \\
\text { organización } \\
\text { contenidos }\end{array}$} & \multirow[t]{3}{*}{ Difusión de contenidos } & $\begin{array}{l}\text { Ad hoc } \\
\text { crossmedia }\end{array}$ & 1 \\
\hline & & Ad hoc & 0,5 \\
\hline & & Crossmedia & 0,5 \\
\hline & \multirow[t]{4}{*}{ Recursos de difusión } & $\begin{array}{l}\text { Directos } \\
\text { repositorio }\end{array}$ & 1 \\
\hline & & Directos & 0,75 \\
\hline & & Repositorio & 0,25 \\
\hline & & Podcasts & 0,25 \\
\hline & \multirow{2}{*}{$\begin{array}{l}\text { Organización de contenidos en } \\
\text { listas }\end{array}$} & Sí & 1 \\
\hline & & No & 0 \\
\hline \multirow{8}{*}{$\begin{array}{l}\text { Narrativas } \\
\text { cocreación }\end{array}$} & \multirow[t]{4}{*}{ Narrativas transmedia } & Sí (>5) & 1 \\
\hline & & Sí (>1) & 0,5 \\
\hline & & Sí (1) & 0,25 \\
\hline & & No & 0 \\
\hline & \multirow[t]{4}{*}{ Espacios para cocreación } & Sí (>5) & 1 \\
\hline & & Sí (>1) & 0,5 \\
\hline & & Sí (1) & 0,25 \\
\hline & & No & 0 \\
\hline
\end{tabular}

Fuente: Elaboración propia 


\section{Resultados}

Todos los medios nativos digitales analizados cuentan con su propio canal de YouTube desde hace como mínimo cinco años (ver tabla 4). El número de seguidores es significativamente diferente entre unos canales y otros, pero se descubre una relación directa entre el número de vídeos subidos y el número de seguidores, de modo que aquellos canales con más seguidores (Eldiario.es, Observador y The Texas Tribune) son también los que más contenido ofrecen a la comunidad, lo que repercute, además, en el número de visualizaciones que alcanzan esos canales.

Tabla 4. Suscriptores, vídeos, visualizaciones y fecha de alta de canales

\begin{tabular}{lllll}
\hline Medio nativo digital & \multicolumn{1}{c}{ Suscriptores } & \multicolumn{1}{c}{ Vídeos } & Visualizaciones & $\begin{array}{c}\text { Fecha } \\
\text { creación }\end{array}$ \\
\hline Eldiario.es & 82.100 & 5.586 & 47.825 .417 & $30 / 05 / 2012$ \\
\hline Les Jours & 415 & 39 & 81.461 & $13 / 03 / 2015$ \\
\hline De Correspondent & 3.230 & 43 & 204.683 & $17 / 03 / 2013$ \\
\hline The Canary & 2.680 & 208 & 303.058 & $13 / 08 / 2015$ \\
\hline Observador & 72.800 & 2.028 & 22.140 .541 & $08 / 05 / 2014$ \\
\hline Krautreporter & 210 & 52 & 8.927 & $26 / 02 / 2015$ \\
\hline The Texas Tribune & 10.900 & 2.527 & 10.539 .711 & $21 / 09 / 2009$ \\
\hline Il Post & No disponible & 90 & 1.851 .980 & $29 / 11 / 2013$ \\
\hline
\end{tabular}

Fuente: YouTube (27/05/2020)

En la consecución de engagement con los públicos, aparte del número de vídeos publicados, influye otro factor directamente relacionado con la cantidad: la frecuencia de publicación. Tomando como referencia el día 27 de mayo de 2020, la fecha de publicación del último vídeo en los respectivos canales se había producido como figura en la tabla 5. Puede observarse que de nuevo hay coincidencia entre los canales con mayor cantidad de suscriptores y los que hacen difusión diaria de contenidos (Eldiario.es y Observador), aunque en el caso de The Texas Tribune la rutina de publicación es semanal. Por el contrario, resulta especialmente llamativo que los demás medios solo publiquen vídeos una vez al mes (Krautreporter) o incluso con una frecuencia que va desde los tres meses al año, lo que evidencia desatención de los canales, más cuando esos mismos diarios realizan difusión diaria de contenidos en Facebook o Twitter.

Tabla 5. Frecuencias de publicación en canales

\begin{tabular}{lll}
\hline Medio nativo digital & \multicolumn{1}{c}{ Último vídeo } & Drecuencia de publicación \\
\hline Eldiario.es & Hoy & - \\
\hline Les Jours & Hace 1 año & - \\
\hline De Correspondent & Hace 3 meses & Semanal \\
\hline The Canary & Hace 2 días & Diaria \\
\hline Observador & Hoy & Mensual \\
\hline Krautreporter & Hace 1 mes & Semanal \\
\hline The Texas Tribune & Hace 1 semana & - \\
\hline Il Post & $\begin{array}{l}\text { Hace 2 semanas* } \\
\text { (El anterior hace 8 meses) }\end{array}$ \\
\hline
\end{tabular}

Fuente: YouTube (27/05/2020)

Con la finalidad de evitar que los canales se conviertan en repositorios de vídeos, desde septiembre de 2016 YouTube ofrece a las marcas una tercera opción para implementar una mejor concepción dialógica e interactiva con los usuarios. Se trata de las comunidades, una iniciativa que permite 
incorporar publicaciones a YouTube, lo que evita que los públicos tengan que recurrir a otras redes sociales para conversar acerca del contenido de los vídeos. Como puede apreciarse en la tabla 6 , son tres los diarios nativos digitales que han incorporado esta opción en sus canales, dos de ellos de los que más suscriptores poseen. No obstante, al analizar las frecuencias de publicación se constata que se conciben como espacios meramente simbólicos, pues tomando como referencia el 27 de mayo de 2020, la fecha de publicación más próxima se sitúa en hace tres semanas (The Canary), mientras que la más lejana es de hace un año (The Texas Tribune).

Tabla 6. Presencias y frecuencias de publicación en comunidades

\begin{tabular}{lcl}
\hline Medio nativo digital & Comunidad & Última actualización \\
\hline Eldiario.es & $\mathrm{X}$ & Hace un mes \\
\hline Les Jours & & \\
\hline De Correspondent & & \\
\hline The Canary & $\mathrm{X}$ & Hace 3 semanas \\
\hline Observador & & \\
\hline Krautreporter & & \\
\hline The Texas Tribune & $\mathrm{X}$ & Hace un año \\
\hline Il Post & & \\
\hline
\end{tabular}

Fuente: YouTube $(27 / 05 / 2020)$

La existencia de estrategias cross-promotion entre la web corporativa y las diferentes plataformas y redes sociales, y viceversa, permite que los públicos puedan navegar de unos espacios digitales a otros, lo que incrementa las posibilidades de interacción y favorece el desarrollo de narrativas transmedia, en el caso de implementarse. Al examinar los widgets de acceso a redes sociales o servicios de mensajería instantánea desde las webs corporativas de los diarios, resulta que solo tres medios (dos de ellos son de los que más suscriptores albergan) ofrecen acceso directo a YouTube. Esta situación provoca que la red social figure en clara desventaja frente a otras redes como Facebook, Twitter o Instagram a pesar de que estas dos últimas cuentan con menos usuarios que YouTube (ver tabla 7).

Tabla 7. Cross-promotion desde web a redes sociales o apps

\begin{tabular}{|c|c|c|c|c|c|c|}
\hline & \multicolumn{5}{|c|}{ Redes sociales } & \multirow{2}{*}{$\begin{array}{c}\text { Mensajería } \\
\text { instantánea } \\
\text { Telegram }\end{array}$} \\
\hline $\begin{array}{l}\text { Medio nativo } \\
\text { digital }\end{array}$ & Facebook & Twitter & Instagram & YouTube & LinkedIn & \\
\hline Eldiario.es & $X$ & $X$ & $X$ & & & $X$ \\
\hline Les Jours & $X$ & $X$ & $X$ & & & \\
\hline$D e$ & $\mathrm{X}$ & $\mathrm{X}$ & $X$ & & $\mathrm{X}$ & \\
\hline \multicolumn{7}{|l|}{ Correspondent } \\
\hline The Canary & $X$ & & $X$ & $X$ & & \\
\hline Observador & $X$ & $X$ & $\mathrm{X}$ & $X$ & $\mathrm{X}$ & \\
\hline \multicolumn{7}{|l|}{ Krautreporter } \\
\hline $\begin{array}{l}\text { The Texas } \\
\text { Tribune }\end{array}$ & $\mathrm{X}$ & $\mathrm{X}$ & $\mathrm{X}$ & $\mathrm{X}$ & $\mathrm{X}$ & \\
\hline Il Post & $X$ & $X$ & & & & $X$ \\
\hline
\end{tabular}

Fuente: Elaboración propia 
Tomando como punto de partida YouTube, las estrategias de promoción cruzada son semejantes, de modo que se constata una preferencia generalizada por dirigir al usuario a Facebook, Twitter e Instagram y también a la web corporativa (ver tabla 8).

Tabla 8. Cross-promotion desde YouTube a web, redes sociales o apps

\begin{tabular}{|c|c|c|c|c|c|c|c|c|}
\hline \multirow[b]{2}{*}{$\begin{array}{c}\text { Medio nativo } \\
\text { digital }\end{array}$} & \multicolumn{5}{|c|}{ Redes sociales } & \multicolumn{2}{|c|}{$\begin{array}{c}\text { Distribuidoras de } \\
\text { audio }\end{array}$} & \multirow{2}{*}{$\begin{array}{c}\text { Mensajería } \\
\text { instantánea } \\
\text { Telegram }\end{array}$} \\
\hline & Web & Facebook & Twitter & Instagram & LinkedIn & Soundcloud & Spotify & \\
\hline Eldiario.es & $\mathrm{X}$ & $\mathrm{X}$ & $\mathrm{X}$ & & & & & \\
\hline Les Jours & & $\mathrm{X}$ & $\mathrm{X}$ & & & & & \\
\hline $\begin{array}{l}\text { De } \\
\text { Correspondent }\end{array}$ & $\mathrm{X}$ & $\mathrm{X}$ & $X$ & $\mathrm{X}$ & & $\mathrm{X}$ & $\mathrm{X}$ & \\
\hline The Canary & $X$ & $X$ & $\mathrm{X}$ & & & & & \\
\hline Observador & $X$ & $\mathrm{X}$ & & $\mathrm{X}$ & & & & \\
\hline Krautreporter & $\mathrm{X}$ & & & & & & & \\
\hline $\begin{array}{l}\text { The Texas } \\
\text { Tribune }\end{array}$ & $X$ & $X$ & $X$ & $\mathrm{X}$ & $X$ & & & \\
\hline Il Post & & & & & & & & $X$ \\
\hline
\end{tabular}

Fuente: Elaboración propia

Las estrategias de cross-promotion en YouTube pueden entenderse también como engranajes que conducen al usuario a otros espacios ajenos al grupo de comunicación al que pertenece el medio nativo digital, pero que la dirección editorial considera afines o de interés para los lectores. Salvo Observador e Il Post todos los medios ofrecen a sus públicos la posibilidad de acceder a terceros canales (ver tabla 9) y dos de los medios con más suscriptores en YouTube (Eldiario.es y The Texas Tribune) son también los medios que ofertan más canales a sus seguidores.

Tabla 9. Cross-promotion desde YouTube a canales externos

\begin{tabular}{ll}
\hline Medio nativo digital & $\begin{array}{c}\text { Número de canales } \\
\text { externos }\end{array}$ \\
\hline Eldiario.es & 16 \\
\hline Les Jours & 5 \\
\hline De Correspondent & 1 \\
\hline The Canary & 10 \\
\hline Observador & 0 \\
\hline Krautreporter & 1 \\
\hline The Texas Tribune & 52 \\
\hline Il Post & 0 \\
\hline
\end{tabular}

Fuente: Elaboración propia con datos de YouTube

Con respecto a la arquitectura de la información, los canales de YouTube ofrecen la posibilidad de catalogar los vídeos en listas de reproducción. Todos los medios utilizan esta opción y clasifican los vídeos en función de su temática (entrevistas, debates, eventos, etc.). El análisis de contenido demuestra que salvo The Texas Tribune ningún medio difunde contenido corporativo, sino solo informativo.

No hay, por tanto, preparación de contenidos ad hoc para YouTube, de tal forma que se produce difusión crossmedia de contenidos que se publican en la web y también en otras redes sociales, 
especialmente en Facebook y Twitter. Esta difusión crossmedia que protagoniza los diferentes canales erradica las narrativas transmedia y los espacios de cocreación a pesar de que no son incompatibles per se, sino complementarios. Otra de las potencialidades narrativas que ofrece YouTube, la retransmisión en directo, es utilizada por cuatro medios nativos digitales, de nuevo dos de ellos cuentan con mayor número de suscriptores (ver tabla 10). En el análisis de contenido aflora otro dato sorprendente: la utilización de YouTube para emitir podcasts. Se trata de un recurso que emplean tres medios y que, aunque a veces se presenta con algún tipo de imagen o como grabación de un programa de radio, no suele ser ni el formato ni el tipo de contenido habitual en YouTube.

Tabla 10. Empleo de recursos facilitados por YouTube

\begin{tabular}{lcc}
\hline Medio nativo digital & Directos & Podcasts \\
\hline Eldiario.es & $\mathrm{X}$ & \\
\hline Les Jours & $\mathrm{X}$ & \\
\hline De Correspondent & & \\
\hline The Canary & & \\
\hline Observador & & $\mathrm{X}$ \\
\hline Krautreporter & & $\mathrm{X}$ \\
\hline The Texas Tribune & $\mathrm{X}$ & $\mathrm{X}$ \\
\hline Il Post & $\mathrm{X}$ & \\
\hline
\end{tabular}

Fuente: Elaboración propia

\section{Discusión y conclusiones}

La tabla 11 recoge las puntuaciones que obtiene cada medio en cada uno de los ítems y la puntuación final que consigue. Como puede observarse consiguen más de la mitad (6) de puntos posibles (12) cuatro medios (The Texas Tribune, Eldiario.es, The Canary y Observador -por este orden-) y tres de ellos (The Texas Tribune, Eldiario.es y Observador) son los que también cuentan con mayor número de suscriptores, vídeos y visualizaciones. Queda demostrado, por tanto, que cuanto mayor es el esfuerzo por mantener vivo el canal, cuantas más estrategias de engagement se emplean y cuantos más recursos se utilizan de los que ofrece YouTube, más éxito tienen los canales y más valiosos resultan para los públicos, como así demuestra el número de visualizaciones. Que The Canary no haya alcanzado todavía un número de seguidores tan alto como los otros medios se justifica en que es el más joven de todos, pues surgió en 2015 y se incorporó a YouTube en agosto de ese mismo año (ver tabla 4), cuando otros medios como The Texas Tribune ya contaban con seis años de experiencia en la red social.

Tabla 11. Resultados de evaluación

\begin{tabular}{|c|c|c|c|c|c|c|c|c|c|c|c|c|c|}
\hline \multirow[b]{2}{*}{$\begin{array}{l}\text { Medio } \\
\text { nativo } \\
\text { digital }\end{array}$} & \multirow[b]{2}{*}{$\begin{array}{c}\text { Frec. } \\
\text { public. }\end{array}$} & \multicolumn{3}{|c|}{ Comunidades } & \multicolumn{3}{|c|}{ Cross-promotion } & \multicolumn{3}{|c|}{$\begin{array}{c}\text { Difusión y organización de } \\
\text { contenidos }\end{array}$} & \multicolumn{2}{|c|}{ Narrativas } & \multirow[b]{2}{*}{ Puntos } \\
\hline & & Sí & $\begin{array}{c}\text { Frec. } \\
\text { public. }\end{array}$ & $\begin{array}{c}\text { CP } \\
\text { desde } \\
\text { web }\end{array}$ & $\begin{array}{l}\text { CP desde } \\
\text { YouTube }\end{array}$ & $\begin{array}{c}\text { CP desde } \\
\text { YouTube } \\
\text { a redes }\end{array}$ & $\begin{array}{c}\text { CP } \\
\text { externa }\end{array}$ & $\begin{array}{l}\text { Difusión } \\
\text { contenido }\end{array}$ & Recursos & Listas & Trans & Co & \\
\hline Eldiario & 1 & 1 & 0,1 & 0 & 1 & 1 & 1 & 0,5 & 1 & 1 & 0 & 0 & 7,6 \\
\hline Les Jours & 0 & 0 & 0 & 0 & 0 & 1 & 0,5 & 0,5 & 1 & 1 & 0 & 0 & 4 \\
\hline De Corres. & 0 & 0 & 0 & 0 & 1 & 1 & 0,25 & 0,5 & 0,5 & 1 & 0 & 0 & 4,25 \\
\hline T. Canary & 0,5 & 1 & 0,1 & 1 & 1 & 1 & 0,5 & 0,5 & 0,5 & 1 & 0 & 0 & 7,1 \\
\hline Observ. & 1 & 0 & 0 & 1 & 1 & 1 & 0 & 0,5 & 0,75 & 1 & 0 & 0 & 6,25 \\
\hline Krautrep & 0,1 & 0 & 0 & 0 & 1 & 0 & 0,25 & 0,5 & 0,75 & 1 & 0 & 0 & 3,6 \\
\hline Texas $T$. & 0,5 & 1 & 0 & 1 & 1 & 1 & 1 & 0,5 & 1,25 & 1 & 0 & 0 & 8,25 \\
\hline Il Post & 0 & 0 & 0 & 0 & 0 & 0 & 0 & 0,5 & 1 & 1 & 0 & 0 & 2,5 \\
\hline
\end{tabular}

Fuente: Elaboración propia 
Las principales fortalezas de los medios nativos digitales en YouTube radican precisamente en el modelo de presencia, esto es, en la elección del canal. Se trata de una variable que no se tiene en cuenta en la ficha de análisis del modelo por su falta de alternativa, pues el canal es la única opción factible para las presencias organizacionales en la red social, de modo que cualquier otra posibilidad ya implicaría un punto de origen incorregible que acarrearía consecuencias nefastas para la gestión de la marca.

En segundo lugar, también constituye una fortaleza el empleo de los diferentes recursos o posibilidades de difusión que ofrece YouTube, pues, aunque es cierto que algunos canales funcionan únicamente como repositorios de contenidos difundidos en otros entornos digitales, también se aprovecha la red para la emisión de vídeo en directo e incluso en algunos casos para la transformación de podcasts a formato audiovisual. Por otra parte, la organización de los contenidos en listas de reproducción creadas en función de la temática de los vídeos ayuda al usuario en la búsqueda y la visualización de la información.

En tercer lugar, se aprovecha la oportunidad que ofrecen los escenarios convergentes no solo para realizar una difusión crossmedia de los contenidos, sino que también se explota la cross-promotion para conducir al usuario de la web a las redes sociales y de YouTube a la web y a otras redes sociales, si bien en el primer caso se certifica preferencia por otras redes como Facebook o Instagram frente a YouTube. Además, se diagnostica un tercer modelo de promoción cruzada basada en las suscripciones que los canales realizan a otros y que no coinciden necesariamente con medios pertenecientes al mismo grupo de comunicación, sino también a terceros de temáticas diversas o de ideología afín.

Por otra parte, el análisis deja al descubierto una serie de carencias que podrían pasar desapercibidas para el usuario común, pero que al investigar los canales desde el punto de vista de la investigación académica arrojan un conjunto de deficiencias que al subsanarse mejorarían considerablemente el valor de las presencias de YouTube, tal y como avalan los datos de suscriptores y visualizaciones de los medios que alcanzan las calificaciones mayores.

Se constata, en primer lugar, una desatención absoluta a las comunidades, un espacio habilitado por YouTube para justamente evitar que el diálogo social acerca del contenido audiovisual se trasladase a otras redes. La desatención es notable en cuanto a la habilitación de estos espacios, pero es prácticamente total en cuanto a la frecuencia de publicación de contenidos, pues ningún medio difunde vídeos en la comunidad con frecuencia diaria o semanal. Esto provoca que se reduzca la interacción entre los usuarios de la comunidad y que la transmisión de contenidos adquiera un carácter más unidireccional que dialógico, lo que acrecienta la posibilidad de acabar convirtiendo el canal en un mero repositorio de vídeos, con escaso valor para las audiencias (Waters y Jones, 2011).

El problema de las frecuencias de publicación es especialmente grave en las comunidades, pero no se limita solo a ellas, sino que también en el propio feed se registran casos de medios que no suben vídeos desde hace varios meses o incluso desde hace un año. Entre saturar al usuario (Shay \& Van Der, 2019) y desatenderlo por completo existe un terreno intermedio en el que se sugiere que se sitúen las marcas, de tal forma que una publicación diaria o dos o tres semanales dependiendo del caso pueden ser suficientes para mantener un canal activo que capte la atención de las audiencias. Por el contrario, publicar cada varios meses incide directamente sobre el engagement de los públicos y repercute en el posicionamiento SEO del canal al sufrir un descenso acusado en los resultados de búsquedas. 
Ha de tenerse en cuenta que no todos los medios cuentan con la misma infraestructura ni con los mismos recursos para la producción de contenido audiovisual, pero habría que preguntarse, no obstante, si conviene tener activos canales sin actividad, pues es probable que el daño que producen a la imagen de la marca sea superior a los beneficios, en especial cuando en otras redes como Facebook o Twitter se produce difusión continua y diaria de noticias e informaciones.

Por otro lado, los canales de YouTube no están siendo explotados para la implementación de narrativas transmedia ni de espacios de cocreación. Consideramos que la habilitación de estas opciones conduciría a los medios que mejores resultados obtienen en la evaluación a un nivel de excelencia que incrementaría todavía más su valor para los públicos. De igual modo, en aquellos medios que aprovechan menos los canales de YouTube la utilización de narrativas que obliguen al usuario a completar el storytelling de la web en el canal supondrían un aumento de las visitas y de las visualizaciones. Habilitar espacios para la cocreación o al menos listas de reproducción que alberguen los user generated contents parece una buena alternativa para incentivar la participación de los públicos que, al mismo tiempo, verían incrementado su nivel de compromiso con el medio al entender que ellos también participan en su producción e ideación, siguiendo la línea de las aportaciones de Prahalad y Ramaswamy (2004).

Tampoco se constata la existencia de contenidos creados ad hoc para YouTube, sino que la práctica común es la difusión multiplataforma de un mismo producto que se adapta a los lenguajes específicos de cada sitio digital. Coincidimos con Ma, Tanaka y Nadamoto (2006) en que estas rutinas productivas son las propias de los escenarios convergentes, pero aplicar cross-promotion a la propia difusión crossmedia puede constituir un motor para la generación de leads, por ejemplo promocionando en Facebook contenidos que solo se pueden visualizar en YouTube. En este caso no podríamos referirnos a narrativas transmedia porque el relato no se inicia en una plataforma y se completa en otra, solo se promociona, pero si el impacto es bueno, se convierte en lead y ese lead convierte en modo de tráfico web.

Al igual que varios estudios previos ponen de manifiesto el éxito de los canales de YouTube en la política o la moda (Berrocal, Campos \& Redondo, 2012; Berrocal, Martín \& Gil, 2017; Gil, 2019; Gómez \& López, 2016; Díez, 2017) se anima a los medios de comunicación, en especial a aquellos cuya única presencia es digital, a aprovechar todavía más las posibilidades que ofrece YouTube para el periodismo en un contexto en que, como apuntaron Neuberger, Nuernbergk y Langenohl (2018), empresas y periodistas operan a la vez en varios canales tanto en la producción como en la distribución de noticias. Pero también se entiende que si la marca no tiene capacidad o recursos para nutrir de contenidos el canal, es preferible que se centre en otras redes antes que contar con una presencia mal gestionada o sin actividad.

\section{Referencias}

Artero, J. P. (2010). Online Video Business Models: YouTube vs. Hulu. Palabra Clave, 13(1), 111123. https://doi.org/10.5294/pacla.2010.13.1.7

Asensio, M. D. (2018). Una propuesta metodológica en el aprendizaje de las expresiones idiomáticas en FLE: los vídeos de Youtube y una presentación Power Point. Anales de Filología Francesa, 26, 28-45. https://doi.org/10.6018/analesff.26.1.352301

Battaglini, M. (2015). La figura del Community manager: de la moda a la consolidación Anuncios: Semanario de Publicidad y Marketing, 1512, 34. 
Bazanini, R., Miklos, J., Bazanini, H. L., Dias, F. \& Otelac, F. (2015). Organizational Communication: Relevance of Symbolic Aspects in Strategies Employed By Professional Media Training Relationship Building with Various Stakeholders. Business and Management Review, Special Issue, 4(8), 527-538. https://doi.org/10.1590/1984-9240837

Berrocal, S., Campos, E. \& Redondo, M. (2012). Comunicación Política en Internet: La tendencia al "infoentretenimiento" político en "YouTube". Estudios sobre el mensaje periodístico, 18(2), 643659. https://doi.org/10.5209/rev_ESMP.2012.v18.n2.41037

Berrocal, S., Martín, V. \& Gil, A. (2017). Líderes políticos en YouTube: información y "politainment" en las elecciones generales de 2016 (26J) en España. El profesional de la información, 26(5), 937-946. https://doi.org/10.3145/epi.2017.sep.15

Blanco, S. \& Palomo, B. (2019). Desencuentro de los periodistas con YouTube. El profesional de la información, 28(4). https://doi.org/10.3145/epi.2019.jul.11

Bonsón, E., Bednarova, M. \& Escobar-Rodríguez, T. (2014). Corporate YouTube practices of Eurozone companies. Online Information Review, 38(4), 484-501. http://dx.doi.org/10.1108/OIR$\underline{07-2013-0181}$

Burgess, J. \& Green, J. (2013). YouTube: Online video and participatory culture. John Wiley \& Sons.

Carbajal, T. (2008). El efecto Youtube y las webs españolas de televisión. En: JM. Flores Vivar (Coord.), Blogalaxia y periodismo en la red: estudios, análisis y reflexiones. pp. 505-516. Fragua.

Cobo, C. \& Pardo, H. (2007). Planeta Web 2.0. Inteligencia colectiva o medios fast food. https://universoabierto.org/2016/07/20/planeta-web-2-0-inteligencia-colectiva-o-medios-fastfood/

Costa Sánchez, C. (2016). Las Superbrands y social media en Portugal. Análisis de su estrategia de comunicación en Youtube. Revista Mediterránea de Comunicación, 7(1), 119-135. http://dx.doi.org/10.14198/MEDCOM2016.7.1.7

Costa Sánchez, C. (2014). Audiovisual y Web 2.0. Empleo de YouTube por las empresas españolas con mejor reputación. Chasqui. Revista Latinoamericana de Comunicación, (126), 31- 41. https://doi.org/10.16921/chasqui.v0i126.264

Copple, E. (2012). “Affluencers” by Bravo: Defining an audience through cross-promotion. Popular Communication, 10(4), 286-301. https://doi.org/10.1080/15405702.2012.715327

Curbelo, C. (2015). Información sanitaria en la web 2.0: YouTube como fuente de divulgación del conocimiento sobre lactancia materna [Tesis doctoral. Universitat d'Alacant]. Repositorio Institucional. http://rua.ua.es/dspace/handle/10045/46272

Deltetll, Ll. (2014). Audiencia social versus audiencia creativa: caso de estudio Twitter. Estudios $\begin{array}{llll}\text { sobre el mensaje } & \text { periodístico, } & \text { 33-47. }\end{array}$ http://dx.doi.org/10.5209/rev_ESMP.2014.v20.n1.45217 
Desiderata Revista (2019). RTVE crea un canal en YouTube para dar a conocer y difundir su $\begin{array}{llll}\text { Archivo } & \text { histórico. } & \text { Desiderata, } & \text { 22-23. }\end{array}$ https://dialnet.unirioja.es/servlet/articulo?codigo=7140092

Díez, A. (2017). YouTube como nueva forma de comunicar la moda: Análisis de las youtubers españolas más influyentes. En: A. Larrondo, K. Meso \& S. Peña (coord.), IX Congreso Internacional de Ciberperiodismo: Innovación y emprendimiento al servicio de las audiencias, pp. 140-176. UPV-EHU.

Flores Vivar, J. M. (Coord.) (2008). Blogalaxia y periodismo en la red: estudios, análisis y reflexiones (Artículos, ponencias, comunicaciones y conclusiones del II Congreso Internacional de Blogs y Periodismo en la Red-UCM, 25 y 26 de abril de 2007). Fragua

García, E. (2013). Políticas del activismo mediático en Sud Kivu (R. D. Congo): Mujeres activistas y su uso de blogs, Youtube y Flickr en la Construcción de Paz. Tejuelo: Didáctica de la Lengua y la Literatura. Educación, 8, 124-143.

Gil, M. (2019). ¿Participa la esfera política en YouTube? Producción y consumo de información en la campaña electoral catalana de 2017. Ámbitos: Revista internacional de comunicación, 44, 141161. http://dx.doi.org/10.12795/Ambitos

Gómez, R. \& López, P. (2016). La utilización de YouTube por parte de los principales partidos políticos durante la precampaña de 2015 en España. En: C. Mateos \& F. J. Herrero (Coords.), La pantall insomne. pp. 3120-3136. Sociedad Latina de Comunicación Social

Haridakis, P. \& Hanson, G. (2009). Social interaction and co-viewing with YouTube: Blending mass communication reception and social connection. Journal of Broadcasting \& Electronic Media, 53(2), 317-335. https://doi.org/10.1080/08838150902908270

Hernández, Á., Silva, D. \& Rivera, E. (2013). El community manager: características y funciones básicas. Revista de Psicología y Ciencias del Comportamiento, 4(2), 67-75. https://doi.org/10.1804/rpcc-uacjs.v4i2.57

Hoyos, A. \& Lasso de la Vega, M. C. (2017). Pymes como modelo económico en la creación de estrategias de comunicación. Revista de Ciencias de la Administración y Economía, 13(2), 59-74. https://doi.org/10.17163/ret.n13.2017.04

Igartua, J. J. (2006) Métodos cuantitativos de investigación en Comunicación. Barcelona: Bosch.

Lange, P. G. (2007). Publicly private and privately public: Social networking on YouTube. Journal of Computer Mediated Communication, 13(1), 361-380. https://doi.org/10.1111/j.1083$\underline{6101.2007 .00400 . x}$

Lapowsky, I. (2018). "YouTube debuts plan to promote and fund 'authoritative' news". https://www.wired.com/story/youtube-debuts-plan-to-promote-fund-authoritative-news

López-Roldán, P. \& Fachelli, S. (2015). El diseño de la muestra. En: P. López-Roldán \& S. Fachelli (Eds.) Metodología de la investigación social cuantitativa. Universitat Autònoma de Barcelona. 
Ma, Q., Tanaka, K. \& Nadamoto, A. (2006). Complementary information retrieval for cross-media news content. Information systems, 31(7), 659-678. https://doi.org/10.1016/j.is.2005.12.004

Neuberger, C., Nuernbergk, C. \& Langenohl, S. (2019). Journalism as Multichannel Communication. Journalism Studies, 20 (9), 1260-1280. https://doi.org/10.1080/1461670X.2018.1507685

Otzen, T. \& Manterola, C. (2017). Sampling Techniques on a Population Study. International Journal of Morphology, 35, 227-232. http://dx.doi.org/10.4067/S0717-95022017000100037

Prahalad, C. K. \& Ramaswamy, V. (2004). Co-creation experiences: the next practice in value creation". Journal of interactive marketing, 18 (3), 5-14. https://doi.org/10.1002/dir.20015

Rivera, D. (2012). ¿Qué es transmedia y storytelling? https://agencia.best/blog/transmedia-ystorytelling/

Ruivo, F. \& Gómes-Franco, F. (2019). YouTube como herramienta de refuerzo de marca para la Agencia EFE. Pilares para el éxito en plataformas de vídeos digitales. Hipertext.net: Revista Académica sobre Documentación Digital y Comunicación Interactiva, 18. https://dx.doi.org/10.31009/hipertext.net.2019.i18.04

Salaverría, R., García Avilés, J. A. \& Masip, P. (2010). Concepto de convergencia periodística. En: X. López \& X. Pereira (Coord.). Convergencia Digital. Reconfiguración de los medios de comunicación en España. pp. 41-64. Universidad de Santiago de Compostela.

Sánchez, M. A. (2010). Cultura y comunicación organizacional: Necesario, un enfoque multidisciplinario para el estudio de las empresas. Revista Mexicana de Comunicación, 23(124), 34-35.

Shay, R. \& Van Der, M. (2019). Using Brand Equity to Model ROI for Social Media Marketing. International Journal on Media Management, 21(1), 24-44. https://doi.org/10.1080/14241277.2019.1590838

Sixto, J. (2018). Gestión profesional de redes sociales. Rutinas y estrategias de Community Management. Salamanca: Comunicación Social.

Smith, A. N., Fischer, E. \& Yongjian, C. (2012). How does brand-related user-generated content differ across YouTube, Facebook, and Twitter? Journal of Interactive Marketing, 26(2), 102-113. https://doi.org/10.1016/j.intmar.2012.01.002

Wardle, C. \& Derakhshan, H. (2017). Information disorder: Toward an interdisciplinary framework for research and policy making. Council of Europe Report. https://rm.coe.int/informationdisorder-toward-an-interdisciplinary-framework-for-researc/168076277c

Waters, R. D. \& Jones, P. M. (2011). Using video to build an organization's identity and brand: A content analysis of nonprofit organizations' YouTube videos. Journal of Nonprofit \& Public Sector Marketing, 23(3), 248-268. https://doi.org/10.1080/10495142.2011.594779

Wigley, C. (2013) Dispelling three myths about Likert scales in communication trait research. Communication Research Reports, 366-372. https://doi.org/10.1080/08824096.2013.836937 
Zink, M., Suh, K., Gu, Y. \& Kurose, J. (2008). Watch global, cache local: YouTube network traffic at a campus network: measurements and implications. Electronic Imaging. (R. Rejaie \& R. Zimmermann, Eds.; pp. 681805-681805-681813). https://doi.org/10.1117/12.774903

\section{AUTORES:}

\section{José Sixto-García.}

Profesor de Periodismo en el Departamento de Ciencias de la Comunicación de la Universidad de Santiago de Compostela. Doctor en Comunicación y Periodismo, fue director del Instituto de Medios Sociales (2013-2019) y su investigación se centra en los nuevos medios, las nuevas narrativas y las redes sociales. Pertenece al grupo de investigación Novos Medios [GI-1641 NM]. Sus publicaciones incluyen libros como Fundamentos del marketing digital o Gestión profesional de redes sociales, publicados en editorial de prestigio.

jose.sixto@usc.es

Índice $\mathbf{H}: 13$

Orcid ID: https://orcid.org/0000-0002-2988-0975

Google Scholar: https://scholar.google.es/citations?hl=es\&user=65vIOnQAAAAJ

\section{Ana Isabel Rodríguez-Vázquez.}

Profesora del área de Comunicación Audiovisual en el Departamento de Ciencias de la Comunicación de la Universidad de Santiago de Compostela. Doctora en Periodismo. Trabajó durante más de una década en prensa, televisión e internet (directora de contenido para Galiciadigital.com). Pertenece al Grupo de Investigación en Estudios Audiovisuales [GI-1786]. anaisabel.rodriguez.vazquez@usc.es

Índice $\mathbf{H}: 11$

Orcid ID: https://orcid.org/0000-0001-7975-1402

Google Scholar: https://scholar.google.es/citations?user=pvFnRVEAAAAJ\&hl=es

\section{Xosé Soengas-Pérez.}

Catedrático de Comunicación Audiovisual en el Departamento de Ciencias de la Comunicación de la Universidad de Santiago de Compostela. Sus investigaciones están centradas en el análisis de los contenidos informativos de radio y de televisión. Entre sus últimas publicaciones destacan 'El ciberactivismo en el proceso de cambio político y social en los países árabes' o 'El pluralismo y el control de la información en las televisiones generalistas españolas'.

jose.soengas@usc.es

Índice H: 13

Orcid ID: https://orcid.org/0000-0003-3246-0477

Google Scholar: https://scholar.google.es/citations?user=Rgl8rmgAAAAJ\&hl=es 